FORMATION Formation emploi

Revue française de sciences sociales

134 | Avril-Juin

Pêle-mêle

\title{
La " sécuflexibilité » : au-delà des tensions entre flexibilité et sécurité de l'emploi, les sociétés coopératives et participatives (Scop)
}

"Secuflexibility" : Cooperative and participative societies (Scop), beyond the flexibility-security tension

"Secuflexibility" : die SCOP (Sociétés coopératives et participatives, genossenschaftlicher Unternehmenszusammenschluss) - jenseits der Spannungen zwischen Flexibilität und Sicherheit des Arbeitsplatzes La "seguflexibilidad" : más allá de las tensiones entre flexibilidad y seguridad del empleo, las sociedades cooperativas y participativas (Scop)

Hervé Charmettant, Jean-Yves Juban, Nathalie Magne et Yvan Renou

\section{OpenEdition} Journals

Édition électronique

URL : http://journals.openedition.org/formationemploi/4742

DOI : 10.4000/formationemploi.4742

ISSN : 2107-0946

Éditeur

La Documentation française

Édition imprimée

Date de publication : 21 juin 2016

Pagination : 107-124

ISSN : 0759-6340

\section{Référence électronique}

Hervé Charmettant, Jean-Yves Juban, Nathalie Magne et Yvan Renou, «La « sécuflexibilité » : au-delà des tensions entre flexibilité et sécurité de l'emploi, les sociétés coopératives et participatives (Scop) », Formation emploi [En ligne], 134 | Avril-Juin, mis en ligne le 24 juin 2018, consulté le 30 octobre 2020. URL : http://journals.openedition.org/formationemploi/4742 ; DOI : https://doi.org/10.4000/ formationemploi.4742 


\section{La « sécuflexibilité » : au-delà des tensions entre flexibilité et sécurité de l'emploi, les sociétés coopératives et participatives \\ (Scop)}

Hervé Charmettant Maître de conférences en économie à l'université Grenoble Alpes et membre du CREG (Centre de Recherches en Economie de Grenoble)

JEAN-YVES JUBAN Maître de conférences HDR (habilité à diriger des recherches) en sciences de gestion à I'université Grenoble Alpes, enseignant à l'IUT (Institut universitaire de technologie) de Valence et chercheur au CERAG (Centre d'études et de recherches appliquées à la gestion)

Nathalie Magne Doctorante en sciences économiques au laboratoire Triangle et à l'université Lumière Lyon2

YVAN RENOU Maître de conférences en économie à l'université Grenoble Alpes et chercheur au CREG (Centre de Recherches en Economie de Grenoble)

Résumé

La « sécuflexibilité » : au-delà des tensions entre flexibilité et sécurité de l'emploi, les sociétés coopératives et participatives (Scop)

La "flexicurité " consiste à substituer, d'autres formes de sécurité à celle de l'emploi, remise en cause par l'impératif de flexibilité. Notre étude de terrain montre que dans les Scop (sociétés coopératives et participatives), la logique est renversée ; la sécurité de l'emploi y est en effet prioritaire, la flexibilité provenant de modalités internes, ce qui nous amène à parler de « sécuflexibilité ». Ressortent alors de nos observations, les conditions fortes de cette voie originale et exigeante de conciliation de la sécurité de l'emploi avec la flexibilité, en particulier, la participation des salariés et leur responsabilisation.

Mots clés : sécurisation des parcours professionnels, coopérative de production, sécurité de l'emploi, pratiques de GRH, politique salariale d'entreprise, expression des salaries 
"Secuflexibility": Cooperative and participative societies (Scop), beyond the flexibility-security tension

In a context of prioritized flexibility, "flexicurity" substitutes alternative forms of security to job security for employees. Our survey shows a reverse logic in SCOPs (Cooperative and Participative Societies) as job security is priority whereas flexibility is internal: we use the term "secuflexibility". Our observations shed light on the conditions of this original and demanding approach trying to reconcile job security and flexibility. Specifically, we insist on workers' participation and responsibility.

Keywords: flexicurity, producers' cooperative, job security, HR management practice, company wage policy, employees' right of expression

Journal of Economic Literature: J 54, M 51, M 54, M 55

Traduction : Auteur.e.s

Le terme de flexicurité est entré dans le langage courant dans les années 2000, après être apparu au centre des objectifs de la Stratégie européenne de l'emploi (Commission européenne, 2007 ; CAS, 2008). Les débats autour de la loi travail lui redonnent une grande actualité.

Méda (2009) montre que la flexicurité est envisagée, en Europe, comme la solution à des exigences contradictoires entre la demande de flexibilité des entreprises et celle de sécurité des salariés vis-à-vis de leur emploi, dans un contexte de remise en cause des règles et protections envisagées comme un frein à l'emploi. La sécurité de l'emploi est alors considérée comme anachronique et la flexibilité présentée par les entreprises comme une réponse indispensable au nouveau contexte concurrentiel mondialisé. D’autres formes de sécurité compenseraient alors la déstabilisation de la condition salariale (Wilthagen et Tros, 2004) : la sécurité d'employabilité (la garantie de disposer d'un emploi), la sécurité de revenu et la sécurité de conciliation (possibilité de concilier vie au travail et hors-travail).

L'engouement pour l'approche en termes d'employabilité et pour ces autres formes de sécurité témoigne que la nécessité de flexibilité pour les entreprises - souvent ramenée à la seule variation des effectifs -, s'est imposée comme une évidence. Cette évidence est interrogée par nos constats sur les pratiques de gestion de la main-d'œuvre au cours d'une étude de terrain sur les Scop, portant à l'origine sur les « relations sociales »" ${ }^{1}$ Le statut légal

1. Nous tenons à remercier les dirigeants de Scop qui nous ont reçus pour mener à bien cette enquête, ainsi que l'Urscop (Union régionale des Scop) pour en soutien. Nous remercions également les rapporteurs anonymes dont les remarques nous ont permis d'améliorer ce texte. 
de ce type d'entreprises, marginal dans le tissu productif français, avec 51000 salariés ${ }^{2}$, les différencie des autres entreprises ( $c f$. encadré 1).

Létude menée a porté sur quarante Scop de la région Rhône-Alpes, constituant un échantillon représentatif (voir encadré 2). Des faits saillants dans les modes de gestion du personnel des Scop sont ressortis de nos observations, qui ne correspondaient pas à la logique de la flexicurité, même si l'on observe une grande diversité de situations.

\section{Encadré 1 - Les Scop (sociétés coopératives et participatives)}

Ces entreprises se caractérisent par la détention du capital par les salariés (ils doivent détenir au minimum $51 \%$ des parts sociales et $65 \%$ des voix à l'assemblée générale), par l'application du principe démocratique à l'assemblée générale (une personne = une voix et non une action = une voix, comme c'est le cas dans les entreprises classiques) et par la répartition des bénéfices respectant certaines règles : au moins $25 \%$ en participation (pour tous les salariés, pas seulement les associés), au moins $16 \%$ en réserves obligatoires (réinvestis dans l'entreprise et constituant des réserves impartageables), au maximum $33 \%$ de dividendes.

Le statut légal de SCOP peut être adopté par toute entreprise (Société Anonyme, Société Anonyme à Resposabilité Limité ou Société par Actions Simplifiée) d'au moins deux personnes. Il a été façonné par quatre lois principales, en 1947, 1978, 1992 et 2014. Les Scop ne peuvent être cotées en bourse et la cession des parts sociales ne permet aucune plus-value.

Au sein des 2680 Scop et Scic(*) qui emploient 51000 salariés en France, 53 \% des travailleurs sont associés ( $80 \%$ des travailleurs avec plus de 2 ans d'ancienneté) et les bénéfices sont partagés de la manière suivante : $40 \%$ en participation, $49 \%$ en réserves obligatoires et $11 \%$ en dividendes.

$\left.{ }^{*}\right)$ : Les SCIC, sociétés coopératives d'intérêt collectif, permettent d'étendre le sociétariat non seulement aux salariés mais également aux fournisseurs, bénéficiaires et collectivités locales.

Pour rendre compte de la priorité accordée à la sécurité de l'emploi dans les Scop et l'utilisation d'autres formes de flexibilité, nous proposons le néologisme "sécuflexibilité "; nous le définissons comme l'une des modalités permettant d'articuler de manière cohérente le développement économique de l'entreprise et le développement personnel de ses membres. Elle répond à la dialectique flexibilité-sécurité qui traverse les organisations productives modernes, en mettant l'accent non pas sur la logique du compromis, reposant sur du donnant-donnant entre contractants, mais sur une logique de garantie collective réglée par le triptyque liberté-sécurité-responsabilité et permettant un développement adaptatif soutenable. Inscrite dans un système de contraintes socio-économiques et financières, sa concrétisation opérationnelle est susceptible de rendre viables les organisations coopératives en structurant leur développement autour du collectif.

2. Chiffres publiés sur le site de la CGSCOP $\underline{\text { http://www.les-scop.coop/sites/fr/les-chiffres-cles/ }}$ 
Comment les Scop réussissent-elles à concilier cette stabilité de l'emploi avec les exigences de flexibilité ? Quelles sont les modalités et les conditions d'application de la " sécuflexibilité " ? Telles sont les questions auxquelles nous tenterons de répondre dans cet article.

Nous présenterons d'abord les modalités de cette sécuflexibilité, observées au sein des Scop, avant d'approfondir les conditions de sa soutenabilité, notamment en termes de participation des salariés.

\section{Encadré 2 - Méthodologie}

Après une première phase exploratoire consistant en six entretiens avec des dirigeants de Scop au cours de l'année 2012, un dispositif d'enquête a été mis en place.

Quarante entretiens d'une durée de une à deux heures ont été réalisés auprès de dirigeants ou responsables de ressources humaines de Scop de la région Rhône-Alpes, entre janvier 2013 et septembre 2014.

Le guide d'entretien était sciemment très peu directif afin de laisser les dirigeants développer les thèmes qui leur semblaient les plus pertinents. Les tensions entre sécurité et flexibilité de l'emploi ne constituaient a priori qu'une partie de notre enquête qui portait plus généralement sur les relations sociales. Cependant, les longs échanges auxquels ce thème a donné lieu nous ont poussés à l'approfondir.

Notre échantillon est représentatif de l'ensemble des Scop de la région en termes de secteur, de taille et d'ancienneté des entreprises et de départements.

La Région Rhône-Alpes compte 400 entreprises Scop ou Scic début 2016, dont 24 \% relèvent de l'industrie, $30 \%$ de la construction et $46 \%$ des services. Les TPE (très petites entreprises) représentent les deux tiers de ces entreprises, seules cinq d'entre elles ayant plus de 100 membres. Enfin, la moitié a moins de cinq ans d'existence et une sur sept a plus de vingt ans d'ancienneté. Les entretiens ont été réalisés par un ou deux enquêteurs et intégralement enregistrés et retranscrits. Nous avons également recueilli des informations comptables sur les entreprises sur leur site internet et sur des sites d'informations comptables et financières.

Les précisions sur cette étude sont présentes dans le rapport d'études diffusé lors de la journée organisée en octobre 2015 à Lyon. Cf. http://projetscop.blogspot.fr

\section{La sécuflexibilité : une réponse originale à la tension flexibilité-sécurité}

Dans cette première partie, nous montrons que la priorité accordée à la stabilité des effectifs, au sein des Scop, est intrinsèquement liée à ce statut particulier. Nous observons ensuite que les formes de flexibilité repérées dans les Scop résultent de cette contrainte de sécurité de l'emploi. 


\subsection{La sécurité de l'emploi : un enjeu prioritaire pour les Scop}

Les constats que nous avons dressés sur le terrain montrent que, dans la plupart des Scop, le facteur travail est considéré comme un "facteur quasi fixe" (Oi, 1962).

Ce n'est certes pas un constat limité à ce type d'entreprises. On a ainsi noté, au niveau macroéconomique, un ajustement très imparfait des emplois au cycle économique, en particulier en France lors de la crise de 2008 (Cochard et alii, 2010). Au niveau microéconomique, on sait que les entreprises, en particulier les TPE et les PME, pratiquent une politique de rétention de la main-d'œuvre (Boutary et Havette, 2009 ; Aït Razouk et Bayad, 2010). Les Scop présentent toutefois des spécificités dans leur gestion des effectifs, en lien avec leur statut particulier.

Ainsi, parmi les Scop étudiées, seuls quatre dirigeants mentionnent qu'ils ont procédé à des licenciements économiques. Toutes ces Scop relèvent d'une activité industrielle, avec un seuil de rentabilité élevé qui exige des ajustements d'effectifs rapides en cas de difficultés. D'autres Scop ont pourtant été touchées par la crise sans se résoudre à licencier. Scopimprimerie, par exemple, a vu son chiffre d'affaires baisser de $29 \%$ entre 2008 et 2012. Son dirigeant nous a clairement indiqué qu'il évitait les licenciements, profitant des départs à la retraite pour diminuer les effectifs qui n’ont baissé que de $8 \%$ sur la même période.

Cette forte stabilité de l'emploi se retrouve dans les contrats de travail. Selon les déclarations des dirigeants, le taux moyen de salariés en CDI (contrat à durée indéterminée) par Scop est de 92,5\%. A titre de comparaison, 78 \% environ des salariés du secteur marchand non agricole sont en CDI au niveau national ${ }^{3}$. Les contrats courts sont rares, les CDD (contrat à durée déterminée) et l'intérim servant essentiellement de période d'essai avant une embauche définitive. Certes, il existe des contrats courts, mais circonscrits à certaines activités spécifiques (formation, édition) ou à forte saisonnalité (restauration).

Les Scop semblent se distinguer par l'absence de clivage en leur sein entre un marché primaire et un marché secondaire, ce dernier servant couramment de variable d'ajustement dans les autres entreprises. La "flexibilité quantitative externe " est ainsi quasiment éliminée par la priorité donnée à la stabilité de l'emploi. La « flexibilité externe qualitative ", consistant à faire appel à des sous-traitants ou à externaliser certaines fonctions tertiaires, a également été très peu observée au sein de notre échantillon, sans doute du fait que les caractéristiques des Scop étudiées ne s'y prêtent pas. Mais on peut l'interpréter aussi comme le refus de reporter les contraintes d'ajustement sur d'autres salariés ou de restreindre l'emploi interne :

3. Calculs réalisés par nos soins à partir des chiffres de la Dares (2015). 
Scopmétallurgie ${ }^{4}:$ "On n'a que des CDI. C'est d'ailleurs quelque chose qui nous a coûté cher, hein. Nous n'avons aucune flexibilité. Nous ne sous-traitons quasiment rien."

Cette sécurité de l'emploi, étendue à tous, est revendiquée par les dirigeants, puisque les trois-quarts d'entre eux déclarent préférer les CDI, avec deux types de justifications. La première concerne un objectif de stabilisation des salariés :

Scopétudes: "Si la personne acceptait un CDD, il devait se plaire dans le système d'un emploi plutôt précaire, [...] ne pas chercher vraiment un emploi stable. Ce que je peux respecter, pas de problème. Sauf que chez nous, c'est pas possible. Quand on rentre à Scopétudes en tant que salarié, on est tenu à être associé au bout de trois ans. Donc forcément, il faut simpliquer dès le départ."

Scoppeinture : "On essaye d'intégrer des nouvelles personnes pour prendre le relais. Notre optique, c'est de transmettre. »

Le second type de justifications des dirigeants de Scop interrogés est d'ordre éthique : l'embauche en CDI correspond à un ensemble de valeurs qui convergent vers la prise en compte du bien-être de chacun et le désir de former un collectif soudé. Ce devoir d'apporter des garanties d'emploi aux membres de la Scop rompt bien avec la conception du travail comme facteur de production ajustable :

Scoplangue : "Ensuite [la stagiaire] a été recrutée en CDI, à temps complet, c'était un engagement lié à nos valeurs. "

Cette volonté de stabiliser les membres de la Scop nous est donc apparue de façon flagrante avec une diversité de justifications qui relève des différentes implications de leur statut coopératif.

Tout d'abord, le principe de la "double qualité " - les salariés étant aussi associés et devant détenir majoritairement le capital -, implique que les membres de la Scop y restent durablement. Pour prendre une participation au capital social, il est nécessaire que les salariés possèdent une certaine ancienneté, les statuts prévoyant généralement une année. Cet acte, qui correspond à un engagement fort, tant financier que moral, exige souvent un temps plus long encore pour qu'un lien de confiance se crée et il suppose évidemment le projet de rester dans l'entreprise.

De plus, le départ de porteurs de parts pose des problèmes à la Scop en raison du remboursement de leurs parts ${ }^{5}$, mais aussi aux salariés, ce remboursement devant intervenir

4. Tous les propos cités sont ceux des gérant(e)s des Scop auprès desquel(le)s nous avons enquêté, sauf mention contraire, propos qui ont été entièrement retranscrits. Tous les noms des Scop ont été anonymisés, comme nous nous y étions engagés dans la charte éthique accompagnant l'étude de terrain.

5. L'obligation statutaire, selon laquelle le capital social ne doit pas descendre en dessous de $50 \%$ du maximum atteint par ce capital, crée un enjeu particulier dans le départ de salariés porteurs d'un grand nombre de parts. 
dans un délai de cinq ans, durée pendant laquelle leur mise peut ainsi être bloquée. Cet effet du sociétariat est fort parmi les Scop enquêtées, puisque $79 \%$ des salariés y ont la double qualité. Ensuite, la stabilité des effectifs met en relief la spécificité de ce type d'entreprises, représentée par leur cohésion autour d'un projet collectif dont la réussite dépend en grande partie de l'adhésion du plus grand nombre. Si cette posture est naturelle pour les fondateurs, elle est plus problématique pour des nouveaux membres, un temps de socialisation coopérative leur étant nécessaire, ce qui oriente les pratiques de recrutement (Juban et alii, 2015a). Ainsi, les formes de management (Lainé, 2015) et les modalités des relations sociales, autorégulées et fondées sur la confiance (Charmettant et alii, 2013), entrent en contradiction avec une rotation du personnel.

Enfin, on peut éclairer ces pratiques au sein des Scop par le projet sociopolitique qu'elles poursuivent et qui forme, avec le projet économique, le " double projet " des Scop, selon notre analyse (Charmettant et alii, op. cit.). La création d'emplois durables et leur transmission à de nouvelles générations, participent de l'utilité sociale revendiquée par les dirigeants des Scop.

Le maintien de l'emploi, sur le long terme et pour tous, et la stabilité du personnel sont bien dans les gènes des Scop. Les ajustements aux modifications de leur activité prennent nécessairement des formes adaptées que nous allons maintenant évoquer.

\subsection{Les formes de flexibilité sous contrainte adoptées par les Scop}

\subsubsection{Flexibilité du temps de travail et polyvalence}

La réticence à introduire de la flexibilité par la variation des effectifs internes ou par le recours à des entreprises externes réduit les possibilités d'adaptation des Scop, à court terme - aux fluctuations de l'activité, et à moyen terme - aux transformations structurelles. La flexibilité en leur sein prend logiquement des formes essentiellement internes. Le volume d'heures de travail constitue ainsi une variable d'ajustement utilisée de façon massive dans les Scop, à la hausse surtout mais aussi à la baisse.

Scopédition : "Il n'y a pas de flexibilité autre que d'allonger le temps de travail. "

Scopélectrique : "Aujourd'hui, à l'atelier, ils font $8 \mathrm{~h}$, la semaine prochaine peut-être 7 , la semaine d'après peut-être qu'on va travailler un samedi matin. [...] Le fait qu'on soit en coopérative fait qu'on n'a pas peur de dire aux clients : "si vous accélérez tout d'un coup les commandes, on est là, on est capables de bosser le samedi, on est capable de faire des heures". "

Les modalités de mise en œuvre de cette flexibilité du temps de travail sont multiples : modulation annuelle, heures supplémentaires régies par un compte épargne-temps ou pas, récupération selon des arrangements informels, parfois même bénévolat,... Elles sont révélatrices de l'appel à la responsabilité des salariés ainsi que de la nécessité de leur engagement pour obtenir ces capacités d'adaptation. Des mécanismes de flexibilité fonctionnelle, ou flexibilité qualitative interne, sont aussi à l'œuvre, en particulier à travers un 
souci constant de polyvalence. Ils permettent d'adapter l'organisation du travail aux circonstances et évitent également les risques de sous-utilisation des salariés aux compétences très étroites.

Scopépicerie : "Il y a besoin de polyvalence sur les tâches du quotidien, tout le monde doit pouvoir tout faire. Le but étant que toute personne ait un doublon efficace."

Scopétudes : "Tout le monde a conscience que tout le travail est nécessaire. Tout le monde fait tout."

\subsubsection{Flexibilité par la réduction des coûts et des rémunérations}

L'autre forme principale d'adaptation est celle qui concerne le résultat financier et ses déterminants. La conséquence directe du maintien de l'emploi, en cas de baisse d'activité, est la dégradation du compte de résultat.

Scopimprimerie : Question : "Comment avez-vous géré la diminution d'activité sans baisser l'emploi? "Réponse : "Ça se sent sur le résultat tout simplement. "

La réduction des coûts peut servir parfois d'amortisseur, comme chez Scoptextile, où un report des actions de formation a été décidé pour concentrer les forces sur la production.

Une autre possibilité rencontrée est la recherche d'activité pour justifier le maintien des effectifs, le volume d'activité devenant la variable d'ajustement. Ce n'est évidemment pas possible pour toutes les Scop. Cette quête du chiffre d'affaires est plus adaptée pour celles dont le marché est en croissance et qui n'ont pas encore fait de prospection commerciale systématique, souvent des petites Scop de services techniques.

Scopélectrique : "Il y a beaucoup de jeunes, ces derniers temps, qui sont rentrés. On s'aperçoit qu'ils sont hyper motivés, donc on se dit: "tiens on va aller chercher du boulot pour les garder parce que demain c'est eux qui prendront des responsabilités". "

Les rémunérations sont affectées par ces variations du résultat car elles en dépendent obligatoirement : le statut de Scop exige qu'au moins $25 \%$ de l'excédent soit distribué en participation. Dans les Scop enquêtées, cette part est perçue par tous les salariés, sociétaires ou non et est en moyenne de $40 \%$, ce qui représente souvent l'équivalent d'un treizième mois. De plus, les deux tiers de ces Scop ont conclu des accords d'intéressement et certaines distribuent des primes collectives qui varient en fonction des résultats.

Enfin, la baisse des résultats aboutit aussi à une diminution des dividendes, voire à l'arrêt de leur versement, ce qui concerne cette fois-ci les seuls sociétaires en proportion de leurs parts. A titre d'exemple, et pour montrer l'ampleur des sommes en jeu, chaque salarié de Scopacier a reçu environ 8000 euros en participation et intéressement en 2010, mais rien en 2013 en raison d'une forte dégradation des résultats financiers. Le poids des éléments variables de rémunération est donc sensiblement plus important que dans les entreprises classiques. 


\subsubsection{Flexibilité par la formation et la promotion interne}

La stabilité de l'emploi, à plus longue échéance, suppose des capacités d'adaptation aux besoins de l'entreprise et donc des politiques de formation couplées à l'ouverture de parcours internes susceptibles de motiver les salariés à rester. Cet " investissement sur les gens ", pour reprendre l'expression du dirigeant de Scopimage, est manifeste à travers les témoignages recueillis :

Scopétude : "Il y a quatre ans, tous les ingénieurs ont été envoyés en formation. Donc ça a été très lourd: en termes de temps parce qu'ils étaient à l'extérieur, c'est des formations très longues, et en termes de coût aussi..."

Scopélectrique : "Mais sinon [si les nouveaux recrutés n’ont pas les compétences] c'est une formation, voilà on a du personnel mixte, on a des gens qui ont aucune connaissance électrique, qui sont rentrés chez nous. "

L'importance de la formation et de la promotion interne, dans les Scop, constitue un indice supplémentaire d'une volonté de stabilisation des salariés. S'il est évident que les formations proposées aux salariés correspondent aux besoins de l'entreprise, les dirigeants interrogés insistent toutefois sur le rôle des salariés dans le choix des formations, sur l'orientation vers la polyvalence et sur la prise en compte des projets professionnels des salariés :

Scopétudes : "Si quelqu'un arrive et me dit : "jai un projet cette année en anglais, il faut absolument que j'aie une formation en anglais", il part en formation immédiatement. "

Scopcouture : "On est en train de monter un nouveau projet de formation pour permettre à tous nos salariés d'obtenir un diplôme, un certificat de qualification professionnelle. "

La formation semble bien aller dans le sens d'une relation de long terme entre les salariés et la Scop. Cet horizon explique aussi les formes de flexibilité dans les Scop - plus internes qu'externes et portant essentiellement sur le temps de travail et son organisation, les résultats financiers et donc les rémunérations -, qui s’accordent avec la priorité donnée au maintien de l'emploi.

Mais à quelles conditions est-ce réalisable et avec quelles conséquences ? C'est à un questionnement plus normatif que nous allons maintenant soumettre cette logique de " sécuflexibilité ", comme nous avons choisi de la nommer.

\section{La participation, au cœur des conditions de soutenabilité de la sécuflexibilité}

Nous verrons dans un premier temps que la sécuflexibilité observée dans les Scop est conditionnée à un degré élevé de "participation » des salariés. Dans un second temps, cet arrangement particulier de la flexibilité et de la sécurité sera exploré sous l'angle des tensions générées dans l'échange salarial. 


\subsection{La participation des salariés fonde la « sécuflexibilité »}

La mise en œuvre des capacités d'adaptation nécessaires à la garantie de sécurité de l'emploi repose sur un ensemble de conditions que nous allons détailler à trois niveaux :

Au niveau financier : la "variabilisation » des charges, en particulier celles liées aux rémunérations, doit être importante afin de permettre un ajustement en cas de baisse des recettes. A cette première condition s'ajoute celle de la possibilité de laisser les résultats comptables se dégrader pour amortir les chocs d'activité. C'est d'autant plus efficace que la situation financière de l'entreprise n'est pas dégradée au moment où les difficultés surviennent et que ses fonds propres sont assez élevés pour passer un mauvais cap.

Au niveau productif: l'organisation de la production doit être suffisamment flexible. Cette flexibilité interne passe par la durée du travail et ses possibles modulations, ainsi que par la polyvalence des salariés et une organisation souple.

Au niveau collectif, les contreparties nécessitées par la sécurité de l'emploi, exigées tant des salariés que des personnes intéressées par le résultat financier, doivent être acceptées et reconnues comme justes et nécessaires. La légitimité des modalités de flexibilité est indispensable pour les rendre efficaces face aux chocs d'activité que subit l'entreprise.

Notre analyse de la sécuflexibilité montre que les Scop sont mieux à même de réunir ces conditions que les entreprises dites « classiques». Cette propriété est liée au degré élevé de "participation » qui est constitutif de leur spécificité, sans toutefois leur être exclusif. Pour argumenter dans ce sens, nous reprenons la distinction opérée par le Conseil d'Analyse Stratégique $(2010 ; 2011)$, entre trois dimensions de la "participation des salariés» : la participation aux résultats, à la gestion quotidienne et aux décisions stratégiques.

\subsubsection{Associer les salariés aux résultats}

La première dimension est celle de "l'association aux résultats" (CAS, 2010, p. 2), dont on a vu qu'elle était inscrite dans le statut des Scop et accrue par l'existence d'accords d'intéressement ou la distribution de primes. C'est le gage, pour les Scop, de la faculté à faire fortement varier les rémunérations de façon à maintenir l'emploi en cas de difficultés.

Certes, cette évolution vers des rémunérations réversibles s'observe dans l'ensemble des entreprises françaises (Amossé et alii, 2008), mais le choix des modalités au sein des Scop est important. Ainsi, nous y avons observé (Juban et alii, 2015b) une préférence pour un partage du risque collectif avec très peu de versement de primes individuelles et un calcul souvent égalitaire du montant de la participation, en proportion du temps de travail et donc déconnecté du salaire, pour une part au moins.

Cela est renforcé par la structure des salaires de base dans les Scop, structure orientée par une même volonté d'égalité. En particulier, nous avons observé que les salaires sont en

6. Pour les Scop de notre échantillon, l'échelle des salaires est en moyenne seulement de deux. Ce chiffre est 
moyenne moins élevés que dans les entreprises classiques pour les postes à responsabilité, mais plus élevés en bas de l'échelle. Ainsi, en cas de réduction de la part variable, les salariés percevant les rémunérations les plus faibles sont moins touchés.

Ces caractéristiques typiques des Scop expliquent que la variabilité des rémunérations ait plus de chances d'y être acceptée, en reposant sur le principe de solidarité et en différenciant ses effets selon le niveau de salaire à l'aune d'un critère de justice.

\subsubsection{Associer les salariés à la gestion quotidienne}

La deuxième dimension de la participation des salariés est "l'association à la gestion quotidienne" (CAS, ibid.). Nos observations de terrain montrent effectivement que le " management participatif» est très présent dans les Scop (Charmettant et Juban, 2016).

Il se déploie à travers des pratiques d'appropriation du travail (Bernoux, 2015), passant par l'autonomie accordée dans l'effectuation des tâches, la personnalisation des profils de poste ou la libre organisation du temps de travail. Le management est participatif également car certaines décisions managériales sont prises après consultation, voire même parfois après validation des salariés concernés par la décision. Il peut s'agir, par exemple, de choix d'investissement, de nomination de responsable d'équipe ou de recrutement, comme nous l'avons souvent observé (Juban et alii, 2015a).

Cette forme de management est favorable à la flexibilité productive en raison de la connaissance du processus productif qu'elle tend à diffuser chez tous les membres de la Scop ${ }^{7}$. Ils sont ainsi capables de s'adapter aux circonstances, en priorisant les tâches et en connaissant les besoins d'autres secteurs de l'entreprise. Ces pratiques leur donnent aussi conscience de la nécessité d'adaptations, par exemple celles concernant le temps de travail, ce qui contribue à les rendre acceptables.

L'appropriation du travail est enfin le gage d'une plus grande polyvalence et de compétences élargies, favorable aux initiatives permettant la flexibilité fonctionnelle.

\subsubsection{Associer les salariés aux décisions stratégiques}

La troisième dimension de la participation des salariés est « l'association aux décisions stratégiques " (CAS, ibid.) qui constitue un trait distinctif des Scop, provenant de leur gouvernance démocratique (voir encadré 1). En particulier, la décision concernant la politique de distribution des dividendes permet au résultat financier de jouer un rôle d'amortisseur. Dans une Scop, contrairement à une société de capital classique, les détenteurs des parts sociales ne sont pas principalement préoccupés par le fait de dégager un résultat comptable positif, obligeant à des ajustements douloureux de l'emploi. La préoccupation de

une moyenne non pondérée, calculée à partir des déclarations des dirigeants.

7. Aoki (1984) a montré comment cette circulation de l'information favorise une meilleure prise de décision. 
ces salariés en majorité sociétaires, est le maintien de leur emploi avant celui de leurs dividendes, limités de toute façon par le statut.

On observe également une solidité financière des Scop liée à la politique de mise en réserves, obligatoire pour une part d'au moins $16 \%$ du bénéfice mais souvent supérieure, qui permet aux Scop de traverser les mauvaises années grâce à des fonds propres importants et un faible endettement.

Là encore, le fait que les salariés-sociétaires décident démocratiquement de l'utilisation du résultat est favorable à une telle prudence, puisqu'ils prennent en compte les menaces sur l'emploi, voire sur la survie de l'entreprise.

De plus, comme nous l'avons vu, la priorité accordée au maintien de l'emploi n'est pas neutre et exige des décisions cruciales sur les rémunérations, l'organisation du travail, voire même la stratégie de l'entreprise. Les procédures démocratiques par lesquelles sont prises ces décisions ont plus de chances de les doter d'une forte légitimité. Celle-ci est encore renforcée par le souci des dirigeants, flagrant lors de nos entretiens, d'informer de façon transparente les salariés sur la santé de l'entreprise et les enjeux concernant son avenir. Cette transparence des informations contribue à faire prendre conscience aux membres de la Scop des nécessités d'adaptation face aux changements structurels ou à une mauvaise passe conjoncturelle.

Le degré élevé de participation des salariés dans les Scop, dans toutes ses dimensions, explique donc en grande partie leur capacité d'adaptation, combinée au maintien de l'emploi. Cette combinaison particulière de sécurité et de flexibilité n'est pas sans enjeux concernant l'échange salarial, enjeux que nous allons maintenant aborder.

\subsection{Les tensions dans la mise en œuvre de la « sécuflexibilité »}

Depuis une trentaine d'années, les reconfigurations du travail salarié, donnant lieu à une grande diversité de formes (Everaere, 2014), mettent à mal les fondements normatifs sur lesquels repose ce statut. Le nouveau partage des risques dissout les sécurités établies par le salariat mais peut également libérer l'initiative individuelle vis-à-vis d'une organisation hiérarchique (Dupuy et Larré, 1998 ; Getz et Carney, 2013). Supiot (2002) résume les nouveaux termes dans lesquels se présente l'équilibre de l'échange salarial en écrivant que "le statut du travailleur, qui reposait sur deux pieds - la dépendance et la sécurité en exige alors trois : la liberté, la sécurité et la responsabilité " des salariés vis-à-vis de leur employeur (p. 5). Et il ajoute que les tensions apparaissent "si l'un de ces trois éléments vient à manquer".

La configuration de la relation d'emploi dans les Scop les place en première ligne de ce mouvement. La sécuflexibilité, dont on a décrit les modalités et les conditions de mise en œuvre, doit être examinée maintenant à l'aune de l'équilibre de l'échange salarial tel qu'il se pose dans les nouveaux termes énoncés par Supiot. 
Cette combinaison particulière de sécurité et de flexibilité constitue une sorte de pacte collectif, dont la validité est en rapport avec ce triptyque, caractéristique du fondement normatif de la relation salariale sous ses nouvelles formes. En cela, les Scop sont en quelque sorte des précurseurs, traçant une voie originale et exigeante.

Tout d'abord, s'agissant de la "sécurité ", nous avons vu que celle de l'emploi demeure une priorité au sein des Scop, sans être réservée à une catégorie de salariés. C'est une grande différence avec la dualisation fréquente de l'emploi entre précaires et stables dans les entreprises classiques (CAS, 2009).

On peut affirmer qu'elle s'accompagne aussi d'une sécurité "d'employabilité " dont on peut trouver l'origine dans les pratiques managériales. C'est ce que suggèrent les résultats d'une étude récente relatée par Sigot et Véro (2014), aboutissant à une typologie de "profils d'entreprises liant organisation du travail, GRH et formation ». Parmi les quatre profils dégagés, le profil de "développement des capacités ", caractérisé par un mode d'organisation du travail impliquant fortement les salariés (autonomie, rotation) ainsi qu'une politique de GRH «attentive au développement de l'entreprise... et des salariés» (ibid., p. 2) et un effort de formation très élevé, est celui auquel les auteurs associent la plus grande employabilité des salariés. Or, ces caractéristiques semblent bien se retrouver dans les Scop qui font la part belle à la participation des salariés, ce qui tend à sécuriser ceux-ci dans leur parcours professionnel.

Pour décliner les catégories classiques de Wilthagen et Tros (op. cit.), il faut souligner que la "sécurité de revenu " passe au second plan dans le cadre de la sécuflexibilité, de même que la "sécurité de conciliation» (possibilité de concilier vie au travail et hors-travail). Elles sont, l'une comme l'autre, mises à contribution dans les modalités d'adaptation empruntées par les Scop, ce qui n'est pas sans risque de dérives, comme nous le verrons plus loin. Mais elles sont au cœur de l'exercice de la "responsabilité ", centrale dans la mise en œuvre de cette flexibilité interne. Cette responsabilité, au sens de la nécessité de répondre de ses actes, repose d'abord sur les épaules du dirigeant dans le cas de certaines décisions, en particulier celles du licenciement, touchant aux limites de la sécuflexibilité. Ainsi le dirigeant de Scopmachine nous confie : "Il n'y a pas de délibération dans la décision des licenciements. On peut avoir une discussion sur ce qu'on fait pour poursuivre, mais pas sur le choix des personnes."

Cette " responsabilité " échoit surtout à l'ensemble des membres de la Scop, tenus d'accepter de s'adapter aux conditions économiques. Quant aux effets sur les rémunérations, on a vu que ces dernières reposaient sur des mécanismes favorables à l'instauration de la justice des contreparties exigées par cette modalité d'adaptation. Les adaptations par le temps de travail sollicitent aussi fortement le sens de la responsabilité des membres de la Scop, en exigeant un engagement de leur part qui va au-delà de ce qui est requis dans les entreprises classiques, voire au-delà de ce que le droit du travail autorise, dans le cas par exemple où l'allongement de la durée du travail est de l'ordre du bénévolat. 
Cette responsabilisation fait peser un risque sur la « liberté », que l'on peut définir comme la possibilité, pour les salariés, de choisir leurs conditions d'emploi et de travail. C'est le troisième principe autour duquel l'équilibre des nouvelles configurations du salariat s'établit. Mais les pratiques managériales et surtout la gouvernance démocratique préservent la « liberté » des membres de la Scop, dans le sens où le principe démocratique justifie la limitation des libertés individuelles par la règle du choix de la majorité. Les procédures de choix amenant à établir la sécuflexibilité sont conformes, au sein des Scop, à cette conception de la liberté individuelle, subordonnée à la volonté commune, ce qui suppose que la démocratie soit bien effective ${ }^{8}$.

A travers cette hypothèse, on voit bien que l'équilibre de l'échange salarial n'est pas naturellement réalisé dans les Scop mettant en pratique la sécuflexibilité. De fait, des constats de tension ont bien été observés lors de notre étude et peuvent être caractérisés en termes de déséquilibre dans le triptyque "sécurité-responsabilité-liberté ».

\section{Cas $\mathrm{n}^{\circ} 1$ : liberté et sécurité sans responsabilité}

Le premier cas de déséquilibre apparait dans un cadre où existe une grande autonomie des salariés dans leur travail, couplée à une sécurité de l'emploi et des rémunérations garantie. La conséquence de l'absence de "responsabilité " réside alors parfois dans la faible implication des membres de la Scop dans les adaptations nécessaires.

A titre illustratif, on note des comportements que l'on pourrait qualifier de relativement irresponsables chez Scoptôlerie : l'absence de mobilisation face à la crise traversée par la Scop a remis en cause son résultat financier et sa survie même. On peut l'attribuer à une direction "faible " qui peine à imposer des mesures, faiblesse que ne compense pas une cohésion élevée.

A l'inverse, une solidarité très présente dans certaines Scop peut les conduire à trop retarder la réduction des effectifs. Dans cette situation, l'absence du sens des responsabilités aboutit à préférer « couler ensemble » que de devoir choisir de réduire les effectifs.

\section{Cas $n^{\circ} 2$ : liberté et responsabilité sans sécurité}

Dans ce cas, l'autonomie individuelle du travailleur est combinée à une très forte responsabilisation qui reporte sur chacun les efforts d'adaptation, ce qui signifie une absence de garantie quant au niveau de revenu, des horaires de travail extensibles et très variables et une polyvalence extrême.

8. Or, si le statut Scop implique un minimum de prise de décisions collectives (voir encadré 1), nous avons observé une grande variabilité dans notre échantillon. Le degré d'effectivité de la démocratie n'est pas déterminé simplement par l'adoption du statut de Scop, comme nos observations l'ont bien montré. 
Certaines Scop, celles à petits effectifs, s'apparentant à des associations de travailleurs indépendants, en sont représentatives avec des horaires fluctuants, un salaire lié aux rentrées financières et des activités très indépendantes pour chacun des membres.

Un autre vecteur de cette précarisation de la condition salariale dans les Scop peut être le contournement du droit du travail, par exemple en faisant effectuer des heures bénévoles ou en ne respectant pas le profil du poste de travail.

\section{Cas $\mathrm{n}^{\circ} 3$ : sécurité et responsabilité sans liberté}

Cette fois-ci, les adaptations aux changements de l'environnement économique sont assurées par une flexibilité des horaires et des rémunérations qui montre la responsabilisation des salariés, tout en maintenant une sécurité par la garantie d'emploi.

Le déséquilibre de ce régime salarial est lié à la mise en œuvre coercitive de cette sécuflexibilité qui entraîne une limitation de la liberté individuelle vis-à-vis du fonctionnement collectif. Les salariés se voient imposer une flexibilité de leur temps de travail qui ne tient pas compte des désirs individuels, par exemple de compatibilité avec la vie familiale. Une telle situation peut survenir si le pouvoir a été accaparé par une coalition des membres de la Scop, remettant en cause sa gouvernance démocratique.

Cette brève présentation des enjeux dans la mise en œuvre de la " sécuflexibilité " révèle que ce mode particulier de conciliation de sécurité de l'emploi et de flexibilité est au cœur d'interrogations essentielles concernant l'entreprise et les relations nouées avec ceux qui y travaillent.

\section{Conclusion}

La sécuflexibilité, comme nous avons choisi d'appeler les formes de flexibilité observées au sein des sociétés coopératives et participatives (Scop), désigne une combinaison de priorités accordées au maintien de l'emploi et à l'adaptation par deux modalités principales : d'une part, des arrangements multiformes dans l'organisation du travail, surtout au niveau du temps de travail ; d'autre part, les variations du résultat et des rémunérations. Les liens entre le statut particulier des Scop et leur choix fréquent de pratiques de rétention de l'emploi résident dans un mélange de nécessités : celle de garder des salariés qui sont aussi des porteurs de parts sociales ; celle d'un choix éthique revendiqué par les dirigeants et d'une volonté commune ; celle de poursuivre solidairement un projet collectif. Nous avons mis en évidence les conditions de possibilité de cette " sécuflexibilité ", notamment en termes de participation des salariés à trois niveaux : participation financière aux résultats, à la gestion de l'entreprise et aux décisions stratégiques. Cette participation démocratique garantit un certain consensus autour des décisions d'ajustement et dote l'organisation d'une certaine agilité. Mais elle n'exclut pas toutes les tensions entre "sécurité, liberté et responsabilité ", selon le tryptique sur lequel repose l'équilibre de l'échange salarial pour Supiot (op. cit.). 
Au-delà de l'intérêt d'être mieux renseigné sur les pratiques des Scop, l'analyse menée sur ce type d'entreprises a vocation à éclairer plus largement les enjeux de la flexibilité dans les entreprises. Nos observations montrent une possibilité d'apporter une sécurité de l'emploi à travers des modalités de flexibilité qui sollicitent fortement les salariés : accepter des baisses de rémunération, moduler les horaires de travail, se prêter au jeu de la polyvalence,...

Difficile de ne pas faire le lien avec les " accords de maintien de l'emploi » introduits par la loi de sécurisation de l'emploi de juin 2013, ainsi qu'avec les dispositions sur la prééminence de l'accord d'entreprise envisagées par la loi dite « loi travail » El Khomri, en pleine discussion au moment où nous écrivons. Notre analyse permet également de faire ressortir les deux exigences à satisfaire afin que la responsabilisation des salariés soit opérante et acceptable pour accroître la flexibilité des entreprises :

D’une part, la liberté de choix des salariés doit être préservée, ce que les Scop font à travers le respect du principe démocratique. A cet endroit, il faut s'interroger sur la procédure par laquelle l'accord est obtenu (rôle des syndicats, mode de consultation des salariés) et les options disponibles pour les salariés en cas de refus.

D'autre part, une certaine sécurité doit être apportée en contrepartie du partage du risque que les salariés supportent, les Scop le faisant à travers la préservation de l'emploi. Sigot et Véro (op. cit.) montrent par ailleurs l'implication de l'entreprise dans la sécurité d'employabilité à travers sa politique interne d'organisation du travail, de RH et de formation, ce qui fournit une autre piste.

C'est à ces conditions que sécurité et flexibilité pourront se combiner, au sein des entreprises, selon des formules à la fois efficaces et justes, et que ces reconfigurations de l'échange salarial pourront être considérées comme équilibrées.

\section{Bibliographie}

Aït Razouk A., Bayad M. (2010), « La gestion stratégique des ressources humaines dans les PME françaises : quelle place et quelle évolution?", Revue internationale PME : économie et gestion de la petite et moyenne entreprise, vol. 23, n 2, pp. 131-157.

Amossé T., Bloch-London C., Wolff L. (sous la dir.) (2008), Les relations sociales en entreprise, Editions La Découverte, Paris.

Aoki M. (1984), The cooperative game Theory of the Firm, London: Oxford University Press.

Bernoux P. (2015), Mieux-être au travail : appropriation et reconnaissance, Editions Octarès, Toulouse. 
Boutary M., Havette D. (2009), "PME : peut-on choisir de ne pas délocaliser ? ", Annales des Mines - Gérer et comprendre, n 95, mars, pp. 56-66.

Commission européenne (2007), Vers des principes communs de flexicurité : des emplois plus nombreux et de meilleure qualité en combinant flexibilité et sécurité, Bruxelles.

CAS (2011), «Participation des salariés et performance sociale : de nouveaux enjeux pour les entreprises françaises dans un contexte de sortie de crise ", La Note d'analyse TravailEmploi, $\mathrm{n}^{\circ} 210$, janvier.

CAS (2010), «Accroître la performance économique de l'entreprise en améliorant sa gouvernance et la participation des salariés ", Note de veille, $\mathrm{n}^{\circ} 177$, juin.

CAS (2009), "L'ajustement de l'emploi dans la crise : la flexibilité sans la mobilité ? ", Note de veille, $\mathrm{n}^{\circ} 156$, novembre.

CAS (2008), " «Flexicurité» européenne : où en est la France? ", Note de veille, n 102, juin.

Charmettant H., Juban J.-Y. (2016), « Participation-Cohésion-Autoréflexion : le «Triangle d'or» du management dans les Scop ", Management \& Avenir, octobre, nº 88 (à paraître)

Charmettant H., Juban J.-Y., Magne N., Renou Y., Vallet G. (2013), La qualité des relations sociales au sein des SCOP. Premiers enseignements d'une enquête en Rhône-Alpes, décembre, $90 \mathrm{p}$.

Cochard M., Cornilleau G., Heyer E. (2010), "Les marchés du travail dans la crise ", Economie \& Statistique, n 438-440, pp. 181-204.

DARES (2015), "Emploi, chômage, population active : bilan de l'année 2014 ", Dares Analyse, juillet, $\mathrm{n}^{\circ} 50$.

Dupuy Y., Larré F. (1998), « Entre salariat et travail indépendant : les formes hybrides de mobilisation du travail ", Travail \& Emploi, $\mathrm{n}^{\circ} 77$, avril, pp. 1-14.

Everaere C. (2014), Les emplois atypiques. Quelles réponses au besoin de flexicurité ?, Editions Liaisons sociales, Paris.

Getz I., Carney B. M. (2013), Liberté \& Cie. Quand la liberté des salariés fait le succès des entreprises, Flammarion, Paris.

Juban J.-Y., Charmettant H., Magne N. (2015a), « Les enjeux cruciaux du recrutement pour les organisations hybrides : les enseignements à tirer d'une étude sur les Scop ", Management \& Avenir, décembre, nº 82, pp. 81-101.

Juban J.-Y., Boissin O., Charmettant H., Renou Y. (2015b), « La théorie des incitations en question : politique de rémunération et design organisationnel des Scop ", RIMHE, $\mathrm{n}^{\circ} 17$, mai-juin-juillet, pp. 64-83.

Lainé A. (2015), Coopération et Management, Editions L'Harmattan, Paris. 
Méda D. (2009), «Flexicurité : quel équilibre entre flexibilité et sécurité ? „, Droit Social, $\mathrm{n}^{\circ}$ 78, juillet-août, pp. 763-782.

Oi W. Y. (1962), "Labor as a Quasi-Fixed Factor", Journal of Political Economy, vol. 70, $\mathrm{n}^{\circ}$ 6, pp. 538-555.

Sigot J.-C., Vero J. (2014), « Politiques d'entreprise et sécurisation des parcours : un lien à explorer ", Bref-Céreq, 318, janvier.

Supiot A. (2002), « Entre marché et régulation : les nouvelles régulations sociales assurantielles, une sécurité tout au long de la vie? ", texte introductif au $2^{\text {ime }}$ symposium Francel OIT «L'avenir du travail, de l'emploi et de la dynamique sociale : dynamique du changement et protection des travailleurs", Lyon, 17-18 janvier.

Wilthagen T., Tros F. (2004), "The Concept of «Flexicurity»: A New Approach to Regulating Employment and Labour Markets", Transfer-European Review of Labour and Research, n 10 (2), pp. 166-186. 